\title{
Influence of Lateral Walls on Peristaltic Flow of a Third Grade Fluid in a Rectangular Duct
}

Safia Akram ${ }^{1 *}$, Nadeem $\mathrm{S}^{2}$ and Anwar Hussain ${ }^{2}$

${ }^{1}$ Department of Basics Sciences, MCS, National University of Sciences and Technology, Islamabad 44000, Pakistan

${ }^{2}$ Department of Mathematics, Quaid-i-Azam University 45320, Islamabad 44000 Pakistan

\begin{abstract}
In the present study we have discussed the influence of lateral walls on peristaltic flow of a third grade fluid in a rectangular duct. The mathematical equations of the third grade fluid for the rectangular duct are first modeled and then simplified under the assumptions of long wave length and low Reynolds number approximation. The reduced equations are solved analytically using Homotopy perturbation method and the Eigen function expansion method. The graphical results of the present problem are also discussed to see the effects of various emerging parameters. It is observed that with an increase in third grade parameter the pressure rise, pressure gradient and number of the trapping bolus decreases.
\end{abstract}

Keywords: Peristaltic flow; Rectangular duct; Third grade fluid; Analytical solution

\section{Introduction}

Experimental and theoretical research in peristaltic flows has received increased attention during the past several decades. This is due to importance of this area in biomedical engineering and physiology. Several pertinent area of interest (where the peristaltic flows are functional) are urine transport from kidney to bladder, the movement of chyme in the gastrointestinal tract, fluids in the lymphatic vessels, biles from the gall bladder into the duodenum, the movement of spermatozoa in the ductus efferent of the male reproductive tract, the movement of the ovum in the fallopian tube, circulation of blood in small blood vessels, roller and finger pumps, heart lung machine, blood pump machine and dialysis machine etc. After the pioneering work done by Latham [1], various researchers have discussed the peristaltic flows with different geometries [2-10]. Recently, Subba Reddy et al. [11] have examined the flow of a viscous fluid due to symmetric peristaltic waves propagating on the horizontal sidewalls of a rectangular duct. They pointed out that the peristaltic flow of traditional asymmetric two dimensional channels may not better approximate the motion of intrauterine fluid in a sagittal cross section of the uterus. Therefore, they consider the rectangular duct instead of two dimensional channels. Later on Mandiwalla and Archer [12] extended the idea of Subba Reddy et al. [11] and discussed the influence of slip boundary condition on peristaltic pumping in a rectangular channel. Another area of focus in fluid mechanics is the study of non-Newtonian fluids. Some important examples of non-Newtonian fluids are blood, mustard, mayonnaise, tooth paste, asphalt, lava and ice, mud slides, snow avalanches, flow of plasma, nuclear fuel slurries, flow of nuclear fuel slurries flow of liquid metals and alloys, flow of mercury amalgams and lubrications with heavy oils and greases [13-17]. The flow characteristics of nonNewtonian fluids are quite different from those of Newtonian fluids. There are many models of non-Newtonian fluids which exhibits different flow properties. However, Second and third order fluids are those which exhibits both shear thinning and shear thickening effects [18-22].

The main goal here is to present the peristaltic flow of a third grade fluid in a rectangular duct. The governing equations of third grade fluid are simplified under the assumptions of long wave length and low Reynolds number and then the resulting nonlinear equations with the corresponding boundary conditions of rectangular duct are solved analytically with the help of Homotopy perturbation method and eigen function expansion method. The expressions of pressure rise, pressure gradient and stream functions are plotted and discussed for various physical parameters of interest.

\section{Mathematical Modelling}

Let us consider the peristaltic flow of an incompressible third grade fluid in a duct of rectangular cross section having the channel width $2 \mathrm{~d}$ and height 2a. We are considering the Cartesian coordinates system in such a way that $\mathrm{X}$-axis is taken along the axial direction, $\mathrm{Y}$-axis is taken along the lateral direction and $\mathrm{Z}$-axis is along the vertical direction of a rectangular duct as shown in Figure 1.

The peristaltic waves on the walls are represented as

$$
Z=H(X, t)= \pm a \pm b \cos \left[\frac{2 \pi}{\lambda}(X-c t)\right]
$$

where $a$ and $b$ are the amplitudes of the waves, $\lambda$ is the wave length, $t$ is the velocity of propagation, $X$ is the time and is the direction of wave propagation. The walls parallel to $X Z$ plane remain undisturbed and are not subject to any peristaltic wave motion. We assume that the lateral velocity is zero as there is no change in lateral direction of the duct cross section. Let $(U, 0, W)$ be the velocity for a rectangular duct. The governing equations for the flow problem are

$$
\begin{aligned}
& \frac{\partial U}{\partial X}+\frac{\partial W}{\partial Z}=0 \\
& \rho\left(\frac{\partial U}{\partial t}+U \frac{\partial U}{\partial X}+W \frac{\partial U}{\partial Z}\right)=-\frac{\partial P}{\partial X}+\frac{\partial}{\partial X} S_{X X}+\frac{\partial}{\partial Y} S_{X Y}+\frac{\partial}{\partial Z} S_{X Z},
\end{aligned}
$$

*Corresponding author: Safia Akram, Department of Basics Sciences, MCS National University of Sciences and Technology, Islamabad 44000, Pakistan, Tel: 009251561119; E-mail: safia_akram@yahoo.com

Received February 27, 2014; Accepted March 26, 2014; Published April 03, 2014

Citation: Akram S, Nadeem S, Hussain A (2014) Influence of Lateral Walls on Peristaltic Flow of a Third Grade Fluid in a Rectangular Duct. J Appl Mech Eng 3: 140. doi:10.4172/2168-9873.1000140

Copyright: ( 2014 Akram S, et al. This is an open-access article distributed under the terms of the Creative Commons Attribution License, which permits unrestricted use, distribution, and reproduction in any medium, provided the original author and source are credited. 


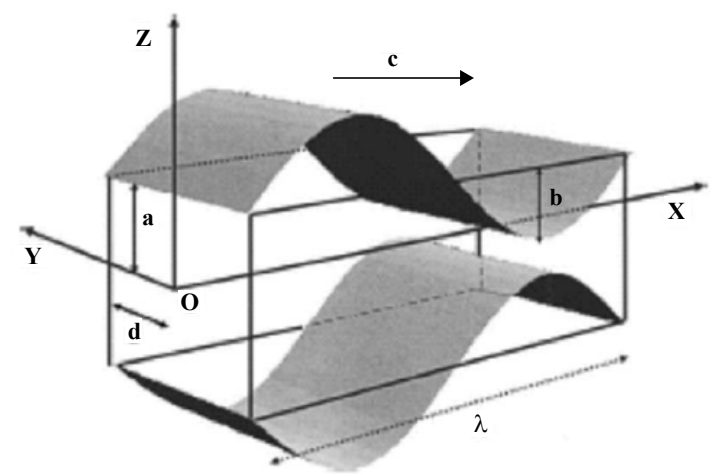

Figure 1: Schematic diagram of peristaltic flow with waves propagating on horizontal walls in a rectangular duct.

$$
\begin{aligned}
& 0=-\frac{\partial P}{\partial Y}+\frac{\partial}{\partial X} S_{Y X}+\frac{\partial}{\partial Y} S_{Y Y}+\frac{\partial}{\partial Z} S_{Y Z}, \\
& \rho\left(\frac{\partial W}{\partial t}+U \frac{\partial W}{\partial X}+W \frac{\partial W}{\partial Z}\right)=-\frac{\partial P}{\partial Z}+\frac{\partial}{\partial X} S_{Z X}+\frac{\partial}{\partial Y} S_{Z Y}+\frac{\partial}{\partial Z} S_{Z Z},
\end{aligned}
$$

in which $\rho$ is the density, $\mathrm{P}$ is the pressure, $\mathrm{t}$ is the time and $S \mathrm{~s}$ is the stress tensor for third grade fluid. The stress tensor for third grade fluid is defined as [23]

$$
\begin{aligned}
& \mathbf{S}=\mu \mathbf{A}_{1}+\alpha_{1} \mathbf{A}_{2}+\alpha_{2} \mathbf{A}_{1}^{2}+\beta_{3}\left(\operatorname{trac} \mathbf{A}_{1}^{2}\right) \mathbf{A}_{1} . \\
& \mathbf{A}_{1}=L+L^{T}, \quad A_{n+1}=\frac{d A_{n}}{d t}+A_{n} L+L^{T} A_{n}
\end{aligned}
$$

Where $L=\operatorname{grad} V, L^{T}=(\operatorname{grad} V)^{T}, \alpha_{1}, \alpha_{2}$ and $\beta_{3}$ are the material moduli.

Let us define a wave frame $(\mathrm{x}, \mathrm{y})$ moving with the velocity $\mathrm{c}$ away from the fixed frame $(\mathrm{X}, \mathrm{Y})$ by the transformation

$$
x=X-c t, y=Y, \quad z=Z, \quad u=U-c, \quad w=W, \quad p(x, z)=P(X, Z, t) .
$$

Defining the following non-dimensional quantities

$$
\begin{aligned}
& \bar{x}=\frac{x}{\lambda}, \bar{y}=\frac{y}{d}, \bar{z}=\frac{z}{a}, \bar{u}=\frac{u}{c}, \bar{w}=\frac{w}{c \delta}, \bar{t}=\frac{c t}{\lambda}, h=\frac{H}{a}, \bar{p}=\frac{a^{2} p}{\mu c \lambda}, \operatorname{Re}=\frac{\rho a c \delta}{\mu}, \\
& \beta=\frac{a}{d}, \delta=\frac{a}{\lambda}, \bar{S}_{\bar{x}}=\frac{a}{\mu c} S_{x x}, \bar{S}_{\bar{y}}=\frac{d}{\mu c} S_{x y}, \bar{S}_{\bar{x}}=\frac{a}{\mu c} S_{x z}, \bar{S}_{\bar{y}}=\frac{d}{\mu c} S_{y z}, \\
& \bar{S}_{\bar{z}}=\frac{\lambda}{\mu c} S_{z z}, \bar{S}_{\bar{y}}=\frac{\lambda}{\mu c} S_{y y}, \lambda_{1}=\frac{\alpha_{1} c}{\mu a}, \lambda_{2}=\frac{\alpha_{2} c}{\mu a}, \Gamma=\frac{\beta_{3} c^{2}}{\mu a^{2}} .
\end{aligned}
$$

Using the above non-dimensional quantities in Equations (2) to (6), the resulting equations after dropping the bars can be written as

$$
\begin{aligned}
& \frac{\partial u}{\partial x}+\frac{\partial w}{\partial z}=0 \\
& \operatorname{Re}\left(u \frac{\partial u}{\partial x}+w \frac{\partial u}{\partial z}\right)=-\frac{\partial p}{\partial x}+\delta \frac{\partial}{\partial x} S_{x x}+\beta^{2} \frac{\partial}{\partial y} S_{x y}+\frac{\partial}{\partial z} S_{x z}, \\
& 0=-\frac{\partial p}{\partial y}+\delta^{2} \frac{\partial}{\partial x} S_{y x}+\delta^{2} \frac{\partial}{\partial y} S_{y y}+\delta \frac{\partial}{\partial z} S_{y z}, \\
& \operatorname{Re} \delta^{2}\left(u \frac{\partial w}{\partial x}+w \frac{\partial w}{\partial z}\right)=-\frac{\partial p}{\partial x}+\delta^{2} \frac{\partial}{\partial x} S_{z x}+\delta \beta^{2} \frac{\partial}{\partial y} S_{z y}+\delta^{2} \frac{\partial}{\partial z} S_{z z},
\end{aligned}
$$

Under the assumption of long eave length $\delta \leq 1$ and Low Reynolds number. Equations. (8) to (11) take the form

$$
\begin{aligned}
\frac{d p}{d x}= & \beta^{2} \frac{\partial^{2} u}{\partial y^{2}}+\frac{\partial^{2} u}{\partial z^{2}}+2 \Gamma \beta^{4} \frac{\partial}{\partial y}\left(\frac{\partial u}{\partial y}\right)^{3}+2 \Gamma \frac{\partial}{\partial z}\left(\frac{\partial u}{\partial z}\right)^{3}+2 \beta^{2} \Gamma \frac{\partial}{\partial y}\left(\frac{\partial u}{\partial y}\left(\frac{\partial u}{\partial z}\right)^{2}\right) \\
& +2 \beta^{2} \Gamma \frac{\partial}{\partial z}\left(\frac{\partial u}{\partial z}\left(\frac{\partial u}{\partial y}\right)^{2}\right)
\end{aligned}
$$

The corresponding boundary conditions are

$$
\begin{aligned}
& u=-1 \text { at } y= \pm 1, \\
& u=-1 \text { at } z= \pm h(x)= \pm 1 \pm \cos 2 \pi x,
\end{aligned}
$$

Where $0 \leq \phi \leq 1, \phi=0$ for straight duct and $\phi=1$ corresponds to total occlusion

\section{Solution of the problem}

Homotopy perturbation method: The Homotopy Perturbation Method for Eq. (12) can be defined as

$$
H(v, q)=(1-\mathrm{q})\left(\mathrm{L}(\mathrm{v})-L\left(u_{0}\right)\right)+q\left(\begin{array}{l}
L(v)+2 \Gamma \beta^{4} \frac{\partial}{\partial y}\left(\frac{\partial u}{\partial y}\right)^{3}+2 \Gamma \frac{\partial}{\partial z}\left(\frac{\partial u}{\partial z}\right)^{3} \\
+2 \beta^{2} \Gamma \frac{\partial}{\partial y}\left(\frac{\partial u}{\partial y}\left(\frac{\partial u}{\partial z}\right)^{2}\right)+2 \beta^{2} \Gamma \frac{\partial}{\partial z}\left(\frac{\partial u}{\partial z}\left(\frac{\partial u}{\partial y}\right)^{2}\right)
\end{array}\right)-\frac{d p}{d x}=0,
$$

Or

$$
\begin{aligned}
H(\mathrm{v}, \mathrm{q})= & \mathrm{L}(\mathrm{v})-\mathrm{L}\left(\mathrm{u}_{0}\right)+q \mathrm{~L}\left(\mathrm{u}_{0}\right)+\mathrm{q}\left(2 \Gamma \beta^{4} \frac{\partial}{\partial y}\left(\frac{\partial u}{\partial y}\right)^{3}+2 \Gamma \frac{\partial}{\partial z}\left(\frac{\partial u}{\partial z}\right)^{3}\right) \\
& +2 \beta^{2} \Gamma \frac{\partial}{\partial y}\left(\frac{\partial u}{\partial y}\left(\frac{\partial u}{\partial z}\right)^{2}\right)+2 \beta^{2} \Gamma \frac{\partial}{\partial z}\left(\frac{\partial u}{\partial z}\left(\frac{\partial u}{\partial y}\right)^{2}\right)-\frac{d p}{d x}=0
\end{aligned}
$$

For our convenience we have taken $L=\beta^{2} \frac{\partial^{2}}{\partial y^{2}}+\frac{\partial^{2}}{\partial x^{2}}$ as the liner operator. We define the initial guess as

$$
u_{0}=-1-h^{2}\left(1-\frac{z^{2}}{h^{2}}-\frac{1}{h^{2} \beta^{2}}\left(1-\mathrm{y}^{2}\right)\right)
$$

Let us define

$$
v(y, z, q)=v_{0}+q v_{1}+q^{2} v_{2}+\ldots
$$

Substituting Equation (17) into Equation (15) and then comparing the like powers of $\mathrm{q}$ one obtains the following problems with the corresponding boundary conditions.

Zeroth order system

$$
\begin{aligned}
& L\left(v_{1}\right)-L\left(u_{0}\right)=0 \\
& v_{0}=-1 \text { at } y= \pm 1, \\
& v_{0}=-1 \text { at } z= \pm h(x),
\end{aligned}
$$

First order system

$$
\begin{aligned}
& L(v)+L\left(u_{0}\right)+\left(2 \Gamma \beta^{4} \frac{\partial}{\partial y}\left(\frac{\partial v_{0}}{\partial y}\right)^{3}+2 \Gamma \frac{\partial}{\partial z}\left(\frac{\partial v_{0}}{\partial z}\right)^{3}\right) \\
& 2 \beta^{2} \Gamma \frac{\partial}{\partial y}\left(\frac{\partial v_{0}}{\partial y}\left(\frac{\partial v_{0}}{\partial z}\right)^{2}\right)+2 \beta^{2} \Gamma \frac{\partial}{\partial z}\left(\frac{\partial v_{0}}{\partial z}\left(\frac{\partial v_{0}}{\partial y}\right)^{2}\right)-\frac{d p}{d x}=0
\end{aligned}
$$


$v_{0}=0$ at $y= \pm 1$,

$v_{0}=0$ at $z= \pm h(x)$,

\section{Solution of Zeroth order system}

With the help of Equation (17) the closed form solution of Equation (19) satisfying the boundary conditions is written as

$$
v_{0}(y, z)=-1-h^{2}(x)\left(1-\frac{z^{2}}{h^{2}(x)}-\frac{1}{h^{2}(x) \beta^{2}}\left(1-y^{2}\right)\right)
$$

Solution of first order system

With the help of Equation. (23), Equation. (21) can be written as

$$
\beta^{2} \frac{\partial^{2} v_{1}}{\partial y^{2}}+\frac{\partial^{2} v_{1}}{\partial z^{2}}=\frac{d p}{d x}+\frac{32 \Gamma}{\beta^{2}} y^{2}-32 \Gamma z^{2}
$$

The solution of the above non-homogeneous partial differential equation can be expressed as

$$
\begin{aligned}
v_{1}(y, z)=\sum_{n=1}^{\infty} & \left(\frac{1}{\cosh \left(\frac{\alpha_{n}}{\beta h(x)}\right)}\left(\frac{2 \beta^{2} b_{0 n}+a_{0 n} \lambda_{n}+\lambda_{n} b_{0 n}}{\lambda_{n}^{2}}\right) \cos \left(\frac{\alpha_{n}}{h(x)} z\right)\right) \\
& -\left(\frac{2 \beta^{2} b_{0 n}+a_{00} \lambda_{n}+\lambda_{n} b_{0 n} y^{2}}{\lambda_{n}^{2}}\right) \cos \left(\frac{\alpha_{n}}{h(x)} z\right)
\end{aligned}
$$

Where

$$
\begin{aligned}
& \alpha_{n}=(2 n-1) \pi / 2 \\
& a_{0 n}=2 \frac{d p}{d x} \frac{(-1)^{n}}{\alpha_{n}}-\frac{64 \Gamma h^{2}(x)(-1)^{n}}{\alpha_{n}}+\frac{128 \Gamma h^{2}(x)(-1)^{n}}{\alpha_{n}^{3}}, \\
& b_{0 n}=\frac{64 \Gamma(-1)^{n}}{\beta^{2} \alpha_{n}} .
\end{aligned}
$$

By using the property of Homotopy perturbation method the original solution can be obtained by using

$$
u(y, z)=\lim _{q \rightarrow 1}\left(v_{0}+q v_{1}+\ldots .\right)
$$

Which is equivalent to

$$
u(y, z)=v_{0}+v_{1}+\ldots . .
$$

Finally, with the help of Equations (23) and (25), Equation (27) can be written as

$$
\begin{aligned}
u(y, z)= & -1-h^{2}(x)\left(1-\frac{z^{2}}{h^{2}(x)}-\frac{1}{h^{2}(x) \beta^{2}}\left(1-y^{2}\right)\right) \\
& +\sum_{n=1}^{\infty}\left(\frac{1}{\cosh \left(\frac{\alpha_{n}}{\beta h(x)}\right)}\left(\frac{\left.2 \beta^{2} b_{0 n}+a_{0 n} \lambda_{n}+\lambda_{n} b_{0 n}\right)}{\lambda_{n}^{2}}\right) \cosh \left(\frac{\alpha_{n}}{\beta h(x)} y\right)\right)- \\
& \left(\frac{2 \beta^{2} b_{0 n}+a_{0 n} \lambda_{n}+\lambda_{n} b_{0 n} y^{2}}{\lambda_{n}^{2}}\right) \cos \left(\frac{\alpha_{n}}{h(x)} z\right)
\end{aligned}
$$

Where constants appearing in Equations (28) are defined Equation (26)

The volumetric flow rate is given by

$$
\begin{aligned}
q & =\int_{0}^{1} \int_{0}^{h(x)} u d y d z \\
= & -h(x)-\frac{2}{3} h^{3}(x)+\frac{2}{3 \beta^{2}} h(x)+2 \frac{d p}{d x} \sum_{n=1}^{\infty} \frac{(-1)^{2 n} h(x)}{\alpha_{n}^{2} \lambda_{n}}\left(-1+\frac{\beta h(x)}{\alpha_{n}} \tanh \left(\frac{\alpha_{n}}{\beta h(x)}\right)\right) \\
& +\sum_{n=1}^{\infty} \frac{\left((-1)^{n} \beta h^{2}(x)\right.}{\alpha_{n}^{2}}\left(\frac{2 \beta^{2} b_{0 n}+\lambda_{n} b_{0 n}}{\lambda_{n}^{2}}-\frac{64 \Gamma(-1)^{n} h^{2}(x)}{\alpha_{n} \lambda_{n}}+\frac{128 \Gamma(-1)^{n} h^{2}(x)}{\alpha_{n}^{3} \lambda_{n}}\right) \tanh \left(\frac{\alpha_{n}}{\beta h(x)}\right) \\
& -\frac{2(-1)^{n} \beta^{2} b_{0 n} h(x)}{\alpha_{n} \lambda_{n}^{2}}-\frac{(-1)^{n} h(x) b_{0 n}}{3 \alpha_{n} \lambda_{n}}-\frac{(-1)^{n} h(x)}{\alpha_{n} \lambda_{n}}\left(-\frac{64 \Gamma(-1)^{n} h^{2}(x)}{\alpha_{n} \lambda_{n}}+\frac{128 \Gamma(-1)^{n} h^{2}(x)}{\alpha_{n}^{3} \lambda_{n}}\right)
\end{aligned}
$$

The instantaneous flux is defined as

$$
\bar{Q}=\int_{0}^{1} \int_{0}^{h(x)}(u+1) d y d z=q+h(x)
$$

The average volume flow rate over one period $\left(T=\frac{\lambda}{c}\right)$ of the peristaltic wave is defines as

$$
Q=\frac{1}{T} \int_{0}^{T} \bar{Q} d t=q+1
$$

The pressure gradient is obtained from Equation (29) and (30) as

$$
\begin{aligned}
& \frac{d p}{d x}=\frac{1}{2 \sum_{n=1}^{\infty} \frac{(-1)^{2 n} h(x)}{\alpha_{n}^{2} \lambda_{n}}\left(-1+\frac{\beta h(x)}{\alpha_{n}} \tanh \left(\frac{\alpha_{n}}{\beta h(x)}\right)\right)}\left(Q-1+h(x)+\frac{2}{3} h^{3}(x)-\frac{2}{3 \beta^{2}} h(x)\right) \\
& -\sum_{n=1}^{\infty}\left(\frac{(-1)^{n} \beta h^{2}(x)}{\alpha_{n}^{2}}\left(\frac{2 \beta^{2} b_{0 n}+\lambda_{n} b_{0 n}}{\lambda_{n}^{2}}-\frac{64 \Gamma(-1)^{n} h^{2}(x)}{\alpha_{n} \lambda_{n}}+\frac{128 \Gamma(-1)^{n} h^{2}(x)}{\alpha_{n}^{3} \lambda_{n}}\right) \tanh \left(\frac{\alpha_{n}}{\beta h(x)}\right)\right) \\
& -\frac{2(-1)^{n} \beta^{2} b_{02} h(x)}{\alpha_{n} \lambda^{2}}-\frac{(-1)^{n} h(x) b_{0 n}}{3 \alpha_{n} \lambda_{n}} \\
& -\frac{(-1)^{n} h(x)}{\alpha_{n} \lambda_{n}}\left(\frac{64 \Gamma(-1)^{n} h^{2}(x)}{\alpha_{n} \lambda_{n}}+\frac{128 \Gamma(-1)^{n} h^{2}(x)}{\alpha_{n}^{3} \lambda_{n}}\right)
\end{aligned}
$$

Integration of Equation (31) over one wavelength yields

$$
\Delta p=\int_{0}^{1} \frac{d p}{d x} d x
$$

Where $d p / d x$ is defined in Equation (31)

It is noticed here that the limit $\beta \rightarrow 0$ (keeping a fixed and $d \rightarrow \infty$ ), the rectangular duct reduces to a two dimensional channel. It is also noticed that when $\beta=1$ the rectangular duct becomes a square duct.

\section{Numerical Result and Discussion}

This section deals with the graphical and numerical results of the

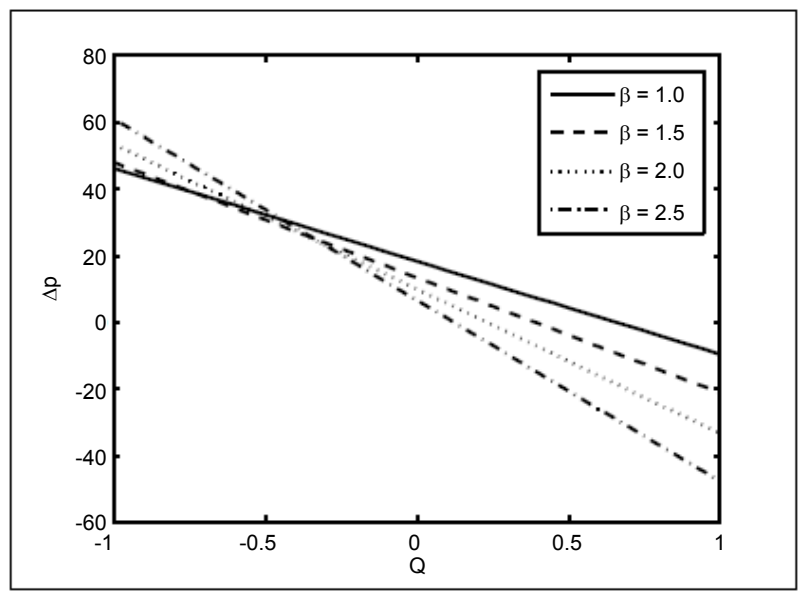

Figure 2: Variation of $\Delta p$ with $Q$ for different values of $\beta$ at $\phi=0.7, \Gamma=0.02$. 


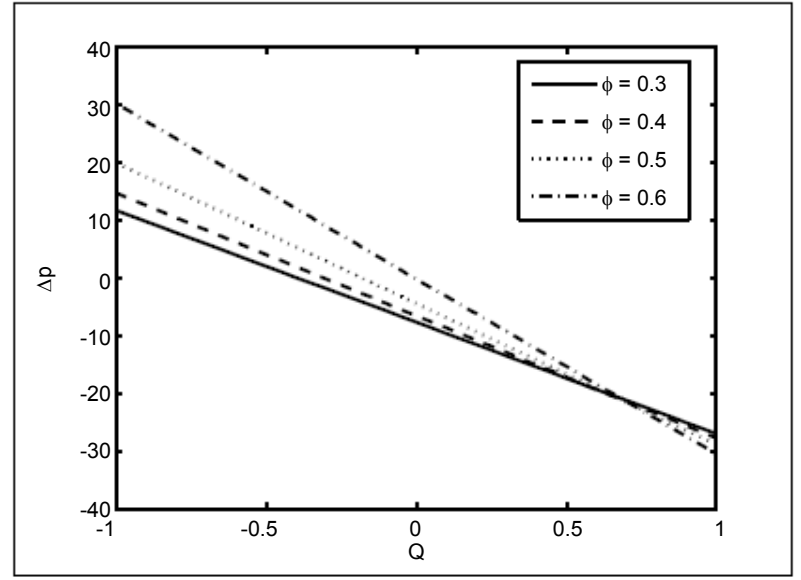

Figure 3: Variation of $\Delta p$ with $Q$ for different values of $\phi$ at $\beta=2.0$ and $\Gamma=0.02$.

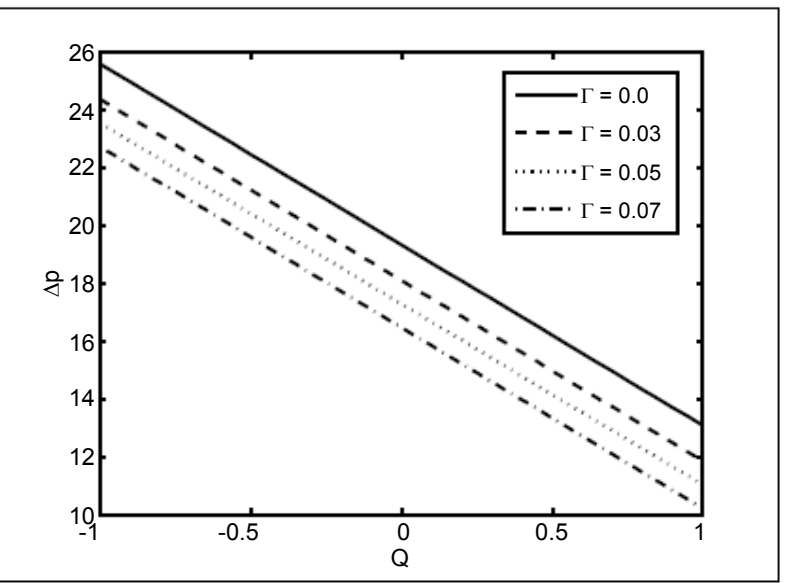

Figure 4: Variation of $\Delta p$ with $Q$ for different values $\Gamma$ at $\beta=0.4$ and $\phi=0.4$.

present problem under discussion. The expression for pressure rise and pressure gradient is calculated numerically using a mathematics software Mathematica. Figures 2-4 show the variation of pressure rise with volume flow rate $\mathrm{Q}$ for different values of aspect ratio $\beta$, amplitude ratio $\phi$, and third grade parameter $\Gamma$. It is observed from Figure 2 that in the retrograde pumping $(\Delta p>0, Q<0)$ region the pressure rise increases with an increase in aspect ratio $\beta$, while in the peristaltic pumping $(\Delta p>0, Q>0)$, (free pumping $\Delta P=0$ ) and copumping $(\Delta p<0, Q<0)$ regions the behavior is quite opposite. Here pressure rise decreases with an increase in aspect ratio $\beta$. Figure 3 shows the variation of pressure rise with volume flow rate $\mathrm{Q}$ for different values of amplitude ratio $\phi$. It is observed that the pressure rise increases with an increase in the retrograde pumping and free regions, while in the copumping region the pressure rise decreases with an increase in amplitude ratio $\phi$. It is observed from Figure 4 that the pressure rise decreases with an increase in third grade parameter $\Gamma$ in all the regions. Figures 5-8 show the variation of the pressure gradient with the space variable $\mathrm{x}$ for different values of aspect ratio $\beta$, amplitude ratio $\phi$, volume flow rate $\mathrm{Q}$ and third grade parameter $\Gamma$. It is depicted that for $x \in[0,0.2]$ and $x \in[0.8,1]$, the pressure gradient is small i.e., the flow can easily pass without imposition of a large pressure gradient, while in the region $x \in[0.2,0.8]$, pressure gradient increases with an increase in

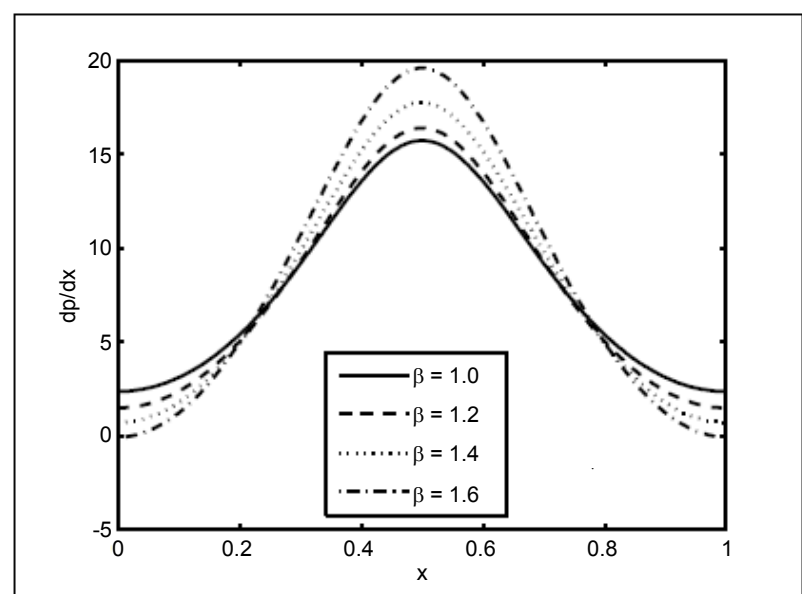

Figure 5: Variation of $d p / d x$ with $\mathrm{x}$ for different values $\beta$ at $\mathrm{Q}=-1, \phi=0.2$ and $\Gamma=0.04$.

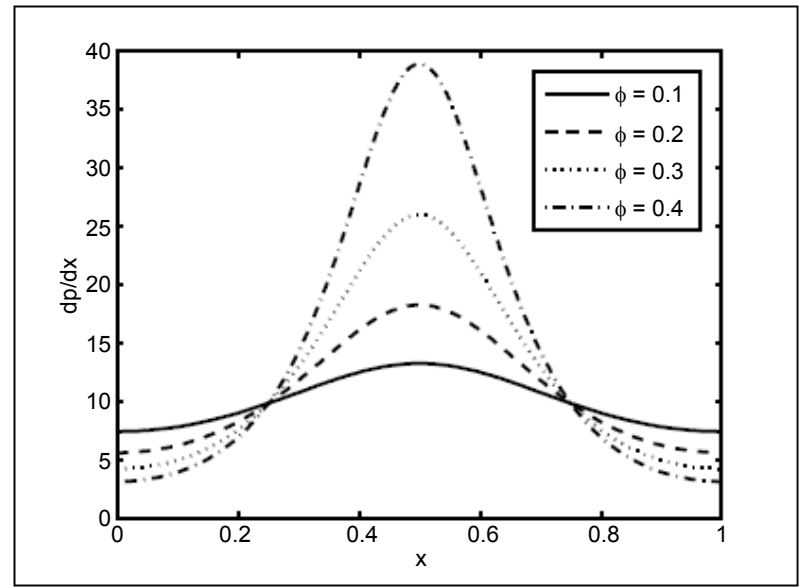

Figure 6: Variation of $d p / d x$ with $\mathrm{x}$ for different values of $\phi$ at $\mathrm{Q}=-0.5, \beta=0.5$ and $\Gamma=0.05$.

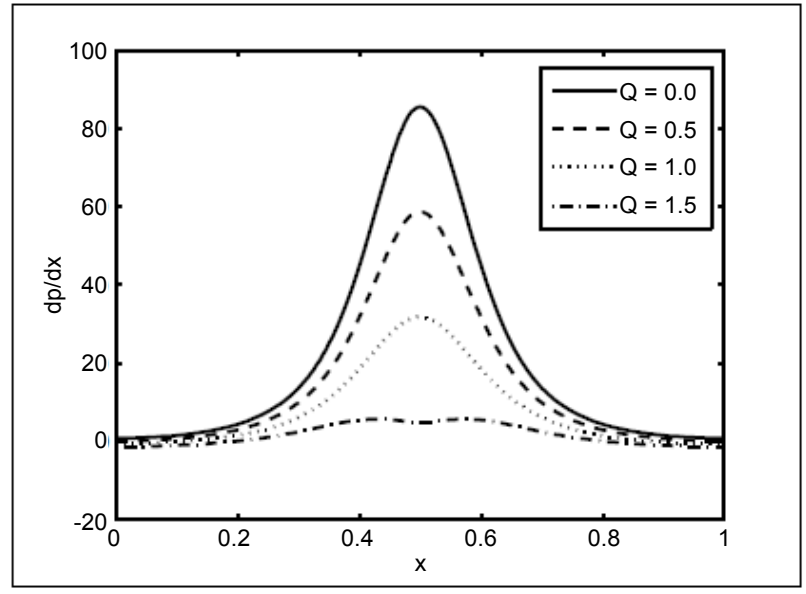

Figure 7: Variation of $d p / d x$ with $x$ for different values of $Q$ at $\beta=0.5, \Gamma=0.05$ and $\phi=0.6$. 


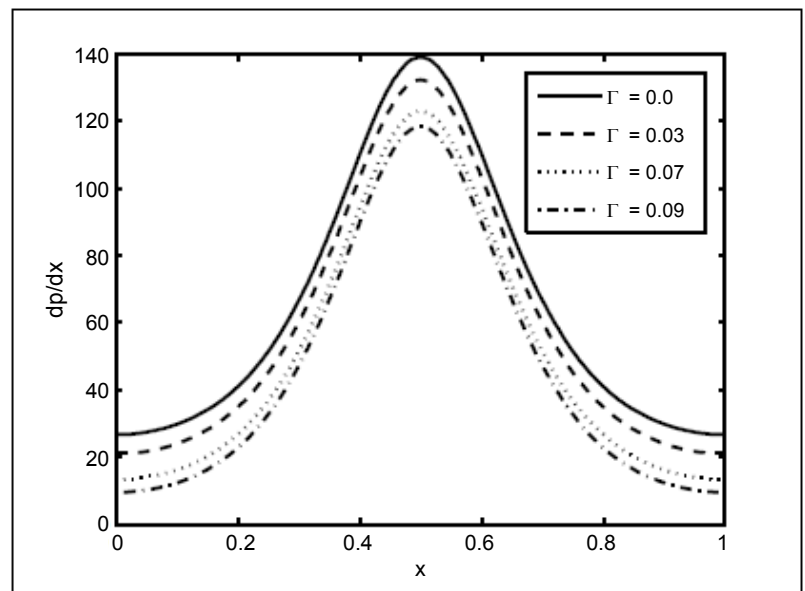

Figure 8: Variation of $d p / d x$ with $\mathrm{x}$ for different values of $\Gamma$ at $\beta=0.2, \mathrm{Q}=1$ and $\phi=0.4$.

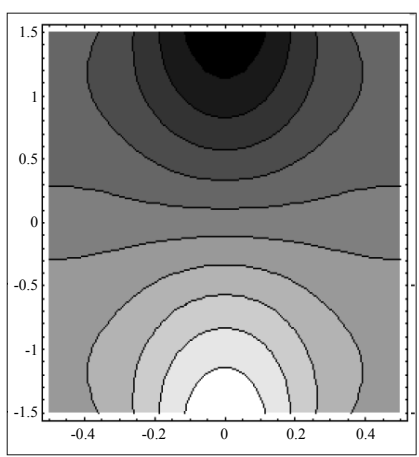

(a)

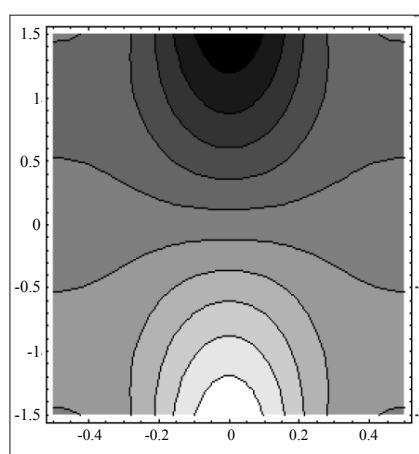

(b)
Figure 9: Streamlines for different values of $\phi$. Figure (a) for $\phi=0.5$, Figure (b) for $\phi=0.8$. for fixed $\beta=0.3, Q=2, \Gamma=0.02$ and $y=1$.

aspect ratio $\phi$ and amplitude ratio $\phi$ and decreases with an increase in volume flow rate $Q$ and third grade parameter $\Gamma$. Figures 9-11 show the streamlines for different values of amplitude ratio $\phi$, aspect ratio $\beta$, Volume flow rate $\mathrm{Q}$ and third grade parameter $\Gamma$. It is observed from Figures 9 and 10 that the size of the trapped bolus decreases with an increase in $\phi, \beta$ and $\mathrm{Q}$, while in the middle of the channel the size of the stream lines increases with an increase in $\phi, \beta$ and $\mathrm{Q}$.

It is observed from Figure 11 that the number of the trapping bolus decreases with an increase in $\Gamma$, while in the middle of the channel the stream lines increases with an increase in $\Gamma$.

\section{Concluding Remarks}

In this paper effect of lateral walls on peristaltic flow of a third grade fluid in a rectangular duct is discussed. Assumptions of long wave length and low Reynolds number approximation is used to develop the simplified mathematical equations of third grade fluid for the rectangular duct. The reduced equations are solved analytically using Homotopy perturbation method and the eigen function expansion method. The results are discussed through graphs. The main findingcan be summarized as follows:

- The pressure rise increases with an increase in aspect ratio $\beta$

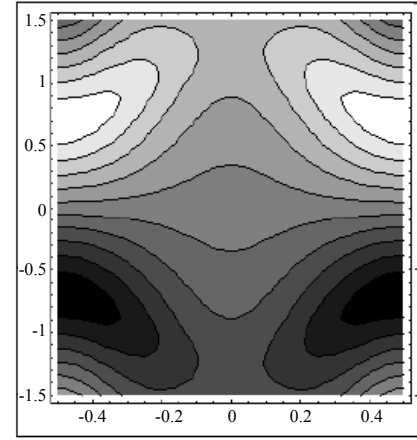

(a)

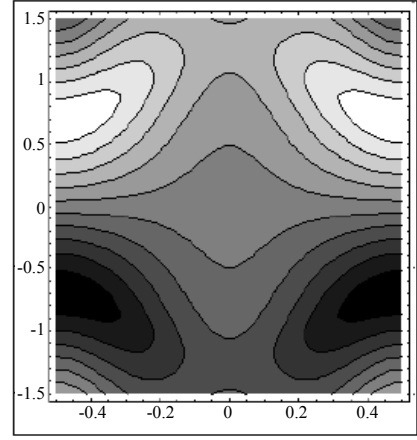

(b)
Figure 10: Streamlines for different values of $\beta$ and Q. Figure (a) for $\beta=0.1$ and $Q=1.5$. Figure $(b)$ for $\beta=0.2$ and $Q=2.5$ for fixed $y=1, \Gamma=0.02$ and $\phi=0.3$.

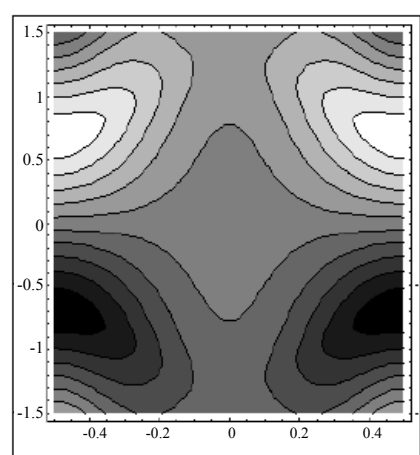

(a)

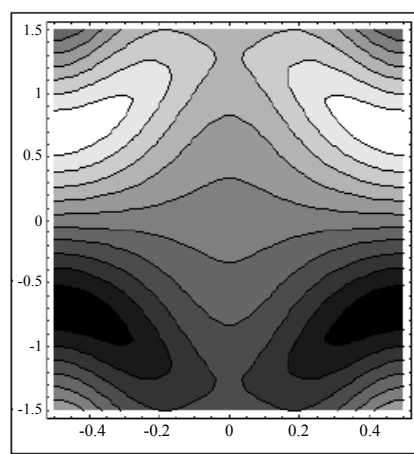

(b)
Figure 11: Streamlines for different values of $\Gamma$. Figure (a) for $\Gamma=0.0$, Figure (b) for $\Gamma=0.05$, fixed $\beta=0.2, Q=1.5, y=1$ and $\phi=0.3$.

in the retrograde pumping region, while in the peristaltic pumping, (free pumping $\Delta p=0$ ) and copumping regions the behavior is quite opposite.

- The pressure rise increases with an increase in amplitude ratio in the retrograde pumping and free pumping regions, while in the copumping region the pressure rise decreases with an increase in amplitude ratio.

- The pressure rise decreases with an increase in third grade parameter $\Gamma$ in all the regions.

- The pressure gradient increases with an increase in aspect ratio $\beta$ and amplitude ratio $\phi$ and decreases with an increase in volume flow rate $\mathrm{Q}$ and third grade parameter $\Gamma$.

- The size of the trapped bolus decreases with an increase in $\phi, \beta$ and $\mathrm{Q}$, while in the middle of the channel the size of the stream lines increases with an increase in $\phi, \beta$ and $\mathrm{Q}$.

- The number of the trapping bolus decreases with an increase in $\Gamma$.

\section{References}

1. Latham TW (1966) Fluid motion in a peristaltic pump, Massachusetts Institute of technology, Cambridge, USA.

2. Weinberg SL (1970) A theoretical and experimental treatment of peristaltic pumping and its relation to ureteral function, Massachusetts Institute of Technology, Massachusetts, USA.

3. Takabatake S, Ayukawa K, Mori A (1988) Peristaltic pumping in circular 
Citation: Akram S, Nadeem S, Hussain A (2014) Influence of Lateral Walls on Peristaltic Flow of a Third Grade Fluid in a Rectangular Duct. J Appl Mech Eng 3: 140. doi:10.4172/2168-9873.1000140

cylindrical tubes: a numerical study of fluid transport and its efficiency, J Fluid Mech 193: 267-283.

4. Mitra TK, Prasad SN (1973) On the influence of wall properties and Poiseuille flow in peristalsis. J Biomechanics 6: 681-693.

5. Nadeem S, Safia Akram (2010) Slip effects on the peristaltic flow of a Jeffrey fluid in an asymmetric channel under the effect of induced magnetic field. Int $\mathrm{J}$ Numer Meth FI 63: 374-394.

6. Nadeem S, Safia Akram (2010) Heat transfer in a peristaltic flow of MHD fluid with partial slip. Commun Nonlinear Sci Numer Simulation 15: 312-321.

7. Ellahi R, Shivanian E, Abbasbandy S, Rahman SU, Hayat T (2012) Analysis of steady flows in viscous fluid with heat transfer and slip effects. Int $\mathrm{J}$ Heat Mass Tran 55: 6384-6390.

8. Ellahi R, Aziz S, Zeeshan A (2013) Non Newtonian nano fluids flow through a porous medium between two coaxial cylinders with heat transfer and variable viscosity. J Porous Media 16: 205-216.

9. Ellahi R (2013) The effects of MHD and temperature dependent viscosity on the flow of non-Newtonian nano fluid in a pipe: analytical solutions. Appl Math Model 37: 1451-1467.

10. Nadeem S, Safia Akram (2010) Peristaltic flow of a Williamson fluid in an asymmetric channel. Commun Nonlinear Sci Numer Simulation 15: 1705-1716.

11. Subba Reddy MV, Mishra M, Sreenadh S, Ramachandra Rao A (2005) Influence of lateral walls on peristaltic flow in a rectangular duct. J Fluid Mech 127: 824-827.

12. Mandiwalla X, Archer R (2008) The influence of slip boundary conditions on peristaltic pumping in a rectangular channel. J Fluid Eng 130.

13. Hajmohammadi MR, Nourazar SS (2014) Conjugate forced convection heat transfer from a heated flat plate of finite thickness and temperature-dependent thermal conductivity. Heat Transfer Eng 35: 863-874.

14. Hajmohammadi MR, Nourazar SS, Ali Manesh H (2012) Semi-analytical treatments of conjugate heat transfer. J Mech Eng Sci 227: 492-503.

15. Hajmohammadi MR, Poozesh S, Rahmani M, Campo A (2013) Heat transfer improvement due to the imposition of non-uniform wall heating for in-tube laminar forced convection. Appl Therm Eng 61: 268-277.

16. Hajmohammadi MR, Campo A, Nourazar SS, Ostad AM (2013) Improvement of forced convection cooling due to the attachment of heat sources to a conducting thick plate. J Heat Trans T 135.

17. Hajmohammadi MR, Salimpour MR, Saber M, Campo A (2013) Detailed analysis for the cooling performance enhancement of a heat source under a thick plate. Energ Convers Manage 76: 691-700.

18. Ellahi R, Afzal S (2009) Effects of variable viscosity in a third grade fluid with porous medium: An analytical solution. Commun Nonlinear Sci Numer Simulation 14: 2056-2072.

19. Ellahi R, Ariel PD, Hayat T, Asghar S (2010) Effect of heat transfer on a third grade fluid in a flat channel. Int J Numer Meth FI 63: 847-859.

20. Ellahi R, Hayat T, Mahomed FM, Asghar S (2010) Effects of slip on the nonlinear flows of a third grade fluid. Nonlinear Anal Series B: Real World Appl 11: $139-146$.

21. Ellahi R, Hayat T, Mahomed FM (2010) Generalized Couette flow of a third grade fluid with slip: the exact solutions. Z Naturforsch A 65a: 1071-1076. 